\title{
ORIGINAL
}

\author{
X. Q. He • C. Qu • Q. H. Qin
}

\section{A theoretical model for surface bone remodeling under electromagnetic loads}

Received: 28 October 2006 / Accepted: 1 May 2007 / Published online: 12 June 2007

(C) Springer-Verlag 2007

\begin{abstract}
A theoretical model for surface bone remodeling under electromagnetic loads is proposed in this paper. In the model, surface bone remodeling is assumed to be related to growth factors. Growth factors in latent form in osteocytes are released to the bone fluid after the osteocytes are absorbed by osteoclasts, and then regulate the bone formation process. At the same time, environmental loadings can influence the generation of growth factors. This paper shows how surface bone remodeling is triggered under the influence of growth factors. Based on this hypothesis, a computational model is established that simulates the bone coupling remodeling process, including internal and surface bone remodeling. The effects of various loadings, including electrical and magnetic loadings, are simulated and compared. The interactions between internal and surface bone remodeling are investigated via the numerical method. The results indicate that an electromagnetic field can strongly influence the bone remodeling process and that the remodeling process will be altered after surface bone remodeling is triggered, compared to the sole effect of the internal remodeling process.
\end{abstract}

Keywords Bone remodeling · Electromagnetic field · Biomaterials · Biomechanics

\section{Introduction}

Interest in the biological effects of extremely low-frequency electromagnetic fields on bone tissues has grown rapidly in recent years [1-3]. Today, increasing numbers of researchers are accepting the notion that there is the potential for interaction between electromagnetic fields and skeletal biological systems. Electromagnetic fields have been widely applied in the treatment of skeletal conditions such as osteoporosis, tendonitis, osteonecrosis, fractures, and nonunion. The influence of an electromagnetic field on bone properties and structures is mostly attributed to its effect on the process of bone functional adaptation. Therefore, the mechanism of how an electromagnetic field acts on bone functional adaptation is an important matter to investigate. Improved understanding of bone cell biology is a key issue in the prevention of osteoporosis and in the improvement of physical and pharmaceutical treatment methods, as well as in prosthetic design.

Functional adaptation of living bone refers to the ability of the tissue to respond to changes in its environment, which is called bone remodeling. Frost redefined bone functional adaptation for cortical bone tissue into two categories: remodeling that involves the turnover of bone in small packets by basic multicellular units

X. Q. He ( $\square)$

Department of Building and Construction, City University of Hong Kong, Hong Kong, China

E-mail: bcxqhe@ cityu.edu.hk

C. Qu

Department of Mechanics, Tianjin University, Tianjin 300072, China

Q. H. Qin

Department of Engineering, Australian National University, Canberra, Australia 
(BMU), and remodeling that refers to the biological processes that produce functionally purposeful sizes and shapes of skeletal organs. These processes mostly involve independent resorption and formation remodeling drifts [4]. Generally, there are two kinds of bone (re)modeling: internal and surface [5]. Internal remodeling (modeling) refers to the resorption or reinforcement of existing bone by decreasing or increasing the bulk density of the bone within fixed external boundaries. Surface remodeling (modeling) refers to the resorption or deposition of bone material on the external surface of the bone. At present, the mechanism by which bone tissues remodel themselves to adapt to environmental loadings is unknown. Many hypotheses and theories have been proposed to explain the relation between bone (re)modeling and its environment [6-11]. Most of these hypotheses deal with mechanical stimuli. However, investigations indicate that electrical signals in bone tissue are of great importance in the bone (re)modeling process [12-17]. It is therefore essential to understand bone (re)modeling behavior under multifield loadings.

The mechanism of how bone cells respond to environmental stimuli is still under investigation. Experimental investigation is an effective and direct method to study bone (re)modeling, but in vivo experiments are hard to control and have a limited life span that is sufficient for the study of only the earliest stages of the adaptive responses. This limited life span is an issue, as the time scale of the relevant processes is on the order of months, or even years. An alternative approach to gathering knowledge of the processes that are involved in bone (re)modeling is computer simulation. In this paper we propose a new theoretical model for surface bone remodeling that is able to predict changes in bone material under the influence of electromagnetic loading. Here, surface remodeling refers to bone surface changes during remodeling. Numerical simulations are conducted based on the proposed model.

\section{Electrical signals in bone remodeling}

Evidence indicates that electrical signals in bone tissue may play an important role in the bone remodeling process. It is commonly believed that the signals that allow bone to adapt to its environment most likely involve strain-mediated fluid flow through the canalicular channels. Fluid can only be moved through bone by cyclic loading, and the flowing fluid generates a streaming potential that is proportional to the strain rate. Active research in the area of tissues such as living bone and collagen has shown that these materials are piezoelectric and that the piezoelectric properties of bone can also enable bone tissue to generate electrical signals that are proportional to the strain rate [18]. All of these factors predict that the magnitude of the adaptive response of bone to loading should be proportional to the strain rate. For lower loading frequencies within the physiological range, experimental evidence shows this to be true. The data also suggest that activities that involve higher loading rates are also effective for increasing bone formation, even if the duration of the activity is short.

To explain the strain-dependent behavior of bone tissues, Frost [19] proposed a mechanostat hypothesis that describes bone (re)modeling, which he updated in [20]. In this model, Frost defined some threshold ranges of the strain-dependent signals (MESm for remodeling and MESr for disuse-mode remodeling. MES here and below stands for minimally effective strains or other stimuli [21]) that help to switch the two whole-bone-strength functions on and off, and showed how these features usually affect bone strength, as illustrated in Fig. 1. The pioneering work of Rubin and Lanyon [22] demonstrated increased activation of remodeling on endocortical and intracortical envelopes when strains were below a remodeling MES of 1,000 microstrains $(\mu \varepsilon)$. Above this threshold, remodeling was inhibited and bone formation was initiated at the periosteal surface, which is consistent with the mechanostat theory. Detailed experiments by Jee et al. indicated that a contralateral overloaded limb showed increased cancellous bone mass due to inhibited remodeling and increased bone formation rate [23]. An increase of cortical bone mass on the periosteum was also observed. How, then, do bone tissues sense strain-related signals, and how are the thresholds distinguished? The foregoing theories do not explain these functions.

As we know, two kinds of bone cells are involved in the bone (re)modeling process, osteoblasts and osteoclasts. The former are responsible for bone formation and the latter for bone resorption. However, evidence has shown that age-related bone osteoporosis differs between males and females. Accelerated bone loss in women during menopause is associated with reduced levels of estrogen. Estrogen receptors are abundantly expressed in osteocytes [24], but their expression is less in other cells of the osteoblast lineage [25], which suggests that osteocytes are likely involved in the regulation of estrogen-mediated bone (re)modeling. Osteocytes can then be considered mechanosensitive bone cells $[26,27]$ that are thought to activate other bone cells to initiate (re)modeling activity in response to environmental stimuli, thus playing a key role in the regulation of bone (re)modeling $[28,29]$. 


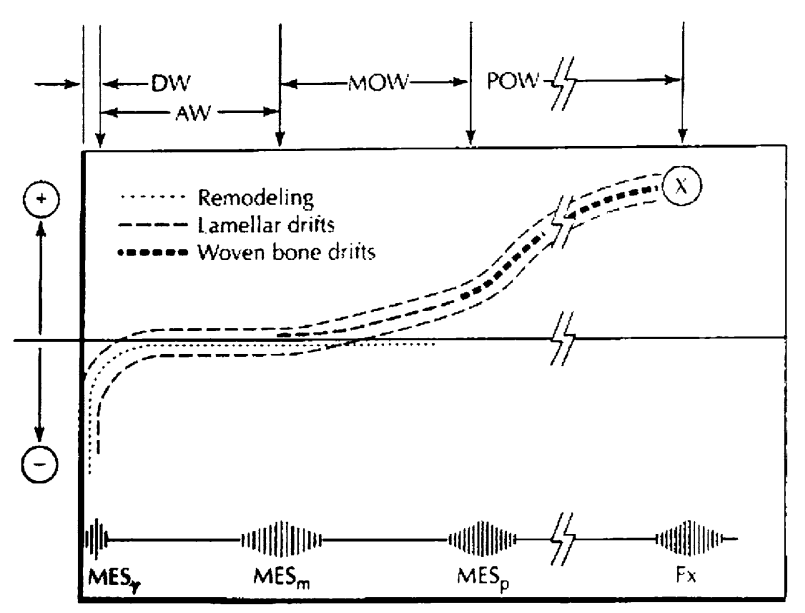

Fig. 1 Combined modeling and remodeling effects on bone strength [19]

How osteocytes participate in (re)modeling is unknown. In former work, a growth-factor-involved hypothesis was proposed to model this process [17]. Growth factors such as platelet-derived growth factor (PDGF), insulin-like growth factor (IGF), bone morphogenetic protein (BMP), and transforming growth factor beta (TGF $\beta$ ) are found in considerable quantities in bone matrix and play an important role in bone formation and (re)modeling [30-33]. Normally they are retained in osteocytes. Once the osteocytes are resorbed, the growth factors are released into the bone fluid and stimulate osteoblasts to refill resorption cavities. Experiments have shown that a pulsed extremely low-frequency electromagnetic field can stimulate the multiplication of growth factors [34-36]. Thus, it can be seen that an electromagnetic field can influence the bone (re)modeling process indirectly.

\section{Theoretical model for bone surface remodeling}

\subsection{Equations of growth and remodeling}

The model proposed in the former work [17] dealt with internal bone (re)modeling. However, some problems still remained unsolved. For example, surface bone remodeling can be observed [23] in the bone remodeling process, but it was not involved in the former model. How then was the surface bone remodeling triggered? It is known that the circular loads that we bear during ordinary daily activities can generate microdamage in our bones, and this can lead to final fracture if not prevented. Hence, the (re)modeling should have a function to repair damage in osteonal bone. However, the generation of microcracks is also strain dependent, and one function of bone (re)modeling is to keep strains in a proper range and ensure less crack production. This can be achieved by the addition of new bone tissue to reduce the porosity of the bone structure and consequently to increase the elastic modulus of bone material, which can decrease the strains on bone structure. The porosity of bone tissue, however, is limited. If the environmental loadings are so great that, even if all of the cavities were filled, the strain on the bone structure could not be reduced to a tolerable level, the bone tissue must do something to prevent this. Another way to reduce the strain is to increase the cross-sectional area, which means decreasing the radius of the inner surface and increasing that of the outer surface. This is defined as surface bone remodeling. In fact, internal remodeling and surface bone remodeling are coupled in the reconstruction of bone material during overload. The interaction process is described as follows. When the bone is overloaded, more growth factors are released into the bone fluid. Osteoblasts are stimulated to deposit more bone material to fill cavities. As the porosity of the bone structure is reduced, the depositing process gradually consumes fewer growth factors. Unconsumed growth factors are transported by the strain-generated fluid flow to the bone surface where they create new osteons.

Based on the foregoing hypothesis, we propose a computable model for the process of surface bone remodeling. In the internal remodeling, we define porosity, $p$, as the measure of bone change. The rate of change of porosity, $\dot{p}$, is then assumed to be a function of the mean bone resorbing $\left(Q_{R}\right)$ and refilling $\left(Q_{F}\right)$ 
rates for each BMU, and the density of resorbing $\left(N_{R}\right)$ and refilling $\left(N_{F}\right)$ BMUs/area [9].

$$
\frac{\mathrm{d} p}{\mathrm{~d} t}=Q_{R} N_{R}-Q_{F} N_{F}
$$

Here, the resorption $\left(Q_{R}\right)$ and refilling $\left(Q_{F}\right)$ rates are assumed to be linear in time. $N_{R}$ contains two parts,

$$
N_{R}=N_{R}^{1}+N_{R}^{0}
$$

where $N_{R}^{0}$ represents the number of BMUs that is required to resorb the naturally timeworn osteocytes and those that have been destroyed by microdamage; and $N_{R}^{1}$ is the population of environmentally stimulated BMUs, which is assumed to be a function of the existing state of damage,

$$
N_{R}^{1}=N_{R(\max )}^{1}\left(1-e^{k_{r} \Phi}\right)
$$

where $N_{R(\max )}^{1}$ is the maximum number of environmentally stimulated BMUs, $N_{R(\max )}^{1}=0.8 \mathrm{BMUs} / \mathrm{mm}^{2}$, and $k_{r}=-1.6$ defines the shape of the curve. $\Phi$ is defined here as the environmental stimulus:

$$
\Phi=C_{i j} \varepsilon_{i j}^{q} R_{L}+\left(C_{i} E_{i}+G_{i} H_{i}\right) f_{e}
$$

Here, $C_{i j}, C_{i}$, and $G_{i}$ are the damage rate coefficients, and $\varepsilon_{i j}, E_{i}$, and $H_{i}$ are the strain, intensity of the electrical field, and intensity of the magnetic field, respectively. The value for the exponent $q$ is set at a nominal value of $\frac{2}{3}$. The mechanical loading rate, $R_{L}$, is assumed to be 3,000 cycles per day (cpd), and $f_{e}$ is the frequency of the electromagnetic field.

The total population $N_{F}^{0}$ is found by multiplying the quantity of resorbed osteocytes by $k_{f}$,

$$
N_{F}^{0}=k_{f} N_{R}
$$

where $k_{f}$ is the correlation coefficient of the refilling BMUs that indicates the relation between the refilling and the resorbing processes. When bone tissues are overloaded, $k_{f}=c_{1}$.

The above model is for internal bone remodeling, detailed analysis of which can be found in the reference [17]. As noted, when the porosity of bone structure is reduced to a certain magnitude, the growth factors exceed the quantities that are consumed by the internal bone remodeling and the excess is transported to the surface of the bone structure, where new bone material is deposited. We define this threshold as $N_{0}$, which is dependent on the porosity $p$. Then we assume a linear relation between the porosity $p$ and the population $N_{0}$ :

$$
N_{0}=k_{s} p
$$

where $k_{s}$ is the proportional coefficient that denotes the maximum growth factors which are consumed by the internal bone remodeling in per unit volume. Because the variation of bone surfaces is very small, the surface bone remodeling rate can be written as

$$
\left\{\begin{array}{l}
-\frac{\mathrm{d} a}{\mathrm{~d} t}=k_{e}\left(N_{F}^{0}-N_{0}\right), \\
\frac{\mathrm{d} b}{\mathrm{~d} t}=k_{p}\left(N_{F}^{0}-N_{0}\right),
\end{array}\right.
$$

where $a$ denotes the radius of the inner surface and $b$ denotes the radius of the outer surface. $k_{e}$ is the coefficient of the endosteal remodeling rate, and $k_{p}$ is the coefficient of the periosteal remodeling rate. At the same time, the population of growth factors to stimulate the internal bone remodeling $N_{F}=N_{0}$. 


\subsection{Equations of piezoelectromagnetism in cylindrical coordinates}

We consider a hollow, circular cylinder of bone that is subjected to a quasistatic axial load $P$, an external pressure $p_{t}$, an electric load $\varphi_{a}$ (and/or $\varphi_{b}$ ) and a magnetic load $\psi_{a}$ (and/or $\psi_{b}$ ). For the sake of simplicity, the cylindrical coordinate system is used in the following analysis. The axial, circumferential, and normal to the middle-surface coordinate length coordinates are denoted by $z, \theta$, and $r$, respectively. In the cylindrical coordinate system, the constitutive equations of a piezoelectromagnetoelastic solid can be given by [16]

$$
\begin{aligned}
& \sigma_{r r}=c_{11} \varepsilon_{r r}+c_{12} \varepsilon_{\theta \theta}+c_{13} \varepsilon_{z z}-e_{31} E_{z}-\alpha_{31} H_{z}, \\
& \sigma_{\theta \theta}=c_{12} \varepsilon_{r r}+c_{11} \varepsilon_{\theta \theta}+c_{13} \varepsilon_{z z}-e_{31} E_{z}-\alpha_{31} H_{z}, \\
& \sigma_{z z}=c_{13} \varepsilon_{r r}+c_{13} \varepsilon_{\theta \theta}+c_{33} \varepsilon_{z z}-e_{33} E_{z}-\alpha_{33} H_{z}, \\
& \sigma_{z r}=c_{44} \varepsilon_{z r}-e_{15} E_{r}-\alpha_{15} H_{r}, \\
& D_{r}=e_{15} \varepsilon_{z r}+\kappa_{1} E_{r}+d_{1} H_{r}, \\
& D_{z}=e_{31}\left(\varepsilon_{r r}+\varepsilon_{\theta \theta}\right)+e_{33} \varepsilon_{z z}+\kappa_{3} E_{z}+d_{3} H_{z}, \\
& B_{r}=\alpha_{15} \varepsilon_{z r}+d_{1} E_{r}+\mu_{1} H_{r}, \\
& B_{z}=\alpha_{31}\left(\varepsilon_{r r}+\varepsilon_{\theta \theta}\right)+\alpha_{33} \varepsilon_{z z}+d_{3} E_{z}+\mu_{3} H_{z},
\end{aligned}
$$

where $\sigma_{i j}, D_{i}$, and $B_{i}$ are components of stress, electrical displacement, and magnetic induction, respectively, $c_{i j}$ is the elastic stiffness; $e_{i j}$ are piezoelectric constants, $\alpha_{i j}$ are piezomagnetic constants, $\kappa_{i}$ are dielectric permitivities, $d_{i}$ are magnetoelectric constants, and $\mu_{i}$ are magnetic permeabilities. The associated strains, electrical field, and magnetic field are respectively related to the displacements $u_{i}$, electric potential $\varphi$, and magnetic potential $\psi$ as

$$
\begin{array}{ll}
\varepsilon_{r r}=u_{r, r}, \quad \varepsilon_{\theta \theta}=\frac{u_{r}}{r}, & \varepsilon_{z z}=u_{z, z} \varepsilon_{z r}=u_{z, r}+u_{r, z}, \\
E_{r}=-\varphi_{, r} E_{z}=-\varphi_{, z}, & H_{r}=-\psi_{, r} H_{z}=-\psi_{, z} .
\end{array}
$$

For quasistationary behavior, in the absence of an electric charge, electrical current, or body force, the set of equations for the piezoelectromagnetic theory of bone is completed by adding the following equations of equilibrium for stress, electric displacement, and magnetic induction to Eqs. (8) and (9):

$$
\begin{aligned}
& \frac{\partial \sigma_{r r}}{\partial r}+\frac{\partial \sigma_{z r}}{\partial z}+\frac{\sigma_{r r}-\sigma_{\theta \theta}}{r}=0, \quad \frac{\partial \sigma_{z r}}{\partial r}+\frac{\partial \sigma_{z z}}{\partial z}+\frac{\sigma_{z r}}{r}=0, \\
& \frac{\partial D_{r}}{\partial r}+\frac{\partial D_{z}}{\partial z}+\frac{D_{r}}{r}=0, \quad \frac{\partial B_{r}}{\partial r}+\frac{\partial B_{z}}{\partial z}+\frac{B_{r}}{r}=0 .
\end{aligned}
$$

The boundary conditions are

$$
\begin{aligned}
& \sigma_{r r}=\sigma_{r \theta}=\sigma_{r z}=0, \quad \varphi=\varphi_{a}, \quad \psi=\psi_{a} \quad \text { at } r=a, \\
& \sigma_{r r}=-p_{t}, \quad \sigma_{r \theta}=\sigma_{r z}=0, \quad \varphi=\varphi_{b}, \quad \psi=\psi_{b} \quad \text { at } r=b,
\end{aligned}
$$

and

$$
\int_{S} \sigma_{z z} \mathrm{~d} S=-P,
$$

where $a$ and $b$ denote, respectively, the inner and outer radii of the bone, and $S$ is the cross-sectional area.

The strain, electrical field, and magnetic field can be obtained by solving the above equations. They are written as follows:

$$
\begin{aligned}
\varepsilon_{r r} & =\frac{1}{F_{3}^{*}}\left(\frac{c_{13} P}{\pi\left(b^{2}-a^{2}\right)}-\frac{c_{33} b^{2} p_{t}}{b^{2}-a^{2}}\right)+\frac{a^{2} b^{2} p_{t}}{r^{2}\left(c_{11}-c_{12}\right)\left(b^{2}-a^{2}\right)}, \\
\varepsilon_{\theta \theta} & =\frac{1}{F_{3}^{*}}\left(\frac{c_{13} P}{\pi\left(b^{2}-a^{2}\right)}-\frac{c_{33} b^{2} p_{t}}{b^{2}-a^{2}}\right)-\frac{a^{2} b^{2} p_{t}}{r^{2}\left(c_{11}-c_{12}\right)\left(b^{2}-a^{2}\right)}, \\
\varepsilon_{z z} & =\frac{1}{F_{3}^{*}}\left[\frac{2 c_{13} p_{t} b^{2}}{b^{2}-a^{2}}-\frac{\left(c_{11}+c_{12}\right) P}{\pi\left(b^{2}-a^{2}\right)}\right], \\
\varepsilon_{r z} & =-\frac{e_{15}\left(\varphi_{b}-\varphi_{a}\right)}{r c_{44} \ln (b / a)}-\frac{\alpha_{15}\left(\psi_{b}-\psi_{a}\right)}{r c_{44} \ln (b / a)}, \quad \text { and } \\
E_{r} & =-\frac{\left(\varphi_{b}-\varphi_{a}\right)}{r \ln (b / a)}, \quad H_{r}=-\frac{\left(\psi_{b}-\psi_{a}\right)}{r \ln (b / a)},
\end{aligned}
$$

where $F_{3}^{*}=c_{33}\left(c_{11}+c_{12}\right)-2 c_{13}^{2}$. 
Once the strains, electrical field, and magnetic field are obtained, then the differential equations (1) and (7) can be rewritten as

$$
\left\{\begin{array}{l}
\frac{\mathrm{d} p}{\mathrm{~d} t}=f_{1}(t, p, a, b), \\
\frac{\mathrm{d} a}{\mathrm{~d} t}=f_{2}(t, p, a, b), \\
\frac{\mathrm{d} b}{\mathrm{~d} t}=f_{3}(t, p, a, b),
\end{array}\right.
$$

where

$$
\begin{aligned}
& f_{1}(t, p, a, b)=Q_{R} N_{R}-Q_{F} N_{F}, \\
& f_{2}(t, p, a, b)=-k_{e}\left(N_{F}-N_{0}\right), \\
& f_{3}(t, p, a, b)=k_{p}\left(N_{F}-N_{0}\right),
\end{aligned}
$$

and then the fourth-order Runge-Kutta integrating process is used to obtain the solution.

To show the results more clearly, the changes of the inner and outer surfaces can be written as the following nondimensional parameters:

$$
\varepsilon=\frac{a}{a_{0}}-1, \quad \eta=\frac{b}{b_{0}}-1 .
$$

Eqs. (14)-(18) show that all of the variables are dependent on $r$. Therefore, the environmental stimulus must also be a function of $r$. Then, as the bone remodels itself, the stimuli of the inner and the outer surfaces vary in the radial direction, which results in the variation of the BMU activation frequencies and the quantity of growth factors in different locations of the bone. However, we assume that the growth factors can be uniformly distributed to the bone structures by means of the fluid flow. The homogenized quantities of the growth factors can be shown as follows:

$$
N_{R}^{1}=\frac{\int_{0}^{2 \pi} \int_{R_{1}}^{R_{2}} N_{R(\max )}^{1}\left(1-e^{k_{r} \Phi}\right) r \mathrm{~d} r \mathrm{~d} \theta}{\pi\left(b^{2}-a^{2}\right)},
$$

where $R_{1}$ denotes the inner radius at which the environmental stimulus exceeds the remodeling threshold and $R_{2}$ denotes the outer radius at which the environmental stimulus exceeds the remodeling threshold. We next simulate the bone surface remodeling process numerically based on this model.

\section{Bone remodeling simulation}

To ensure simplicity, we consider a section of hollow cylindrical bone that is subjected to axial compressive pressure $P$, transverse pressure $p_{t}$, and pulsed electromagnetic loads. We also assume that the strain $s_{i j}$, the electric field $E_{i}$, and the magnetic field $H_{i}$ all return to zero at the end of each load cycle, so that their ranges and peaks are the same. The model is given an initial porosity of $8 \%$. The state variables and constants are shown in Table 1.

As a numerical illustration of the bone surface remodeling process, we consider a femur with $a=25 \mathrm{~mm}$ and $b=35 \mathrm{~mm}$. The elastic moduli were calculated as $c_{i j}=c_{i j}^{0}(1-p)^{n}, e_{i j}=e_{i j}^{0}(1-p)^{n}, \alpha_{i j}=\alpha_{i j}^{0}(1-p)^{n}$, where $c_{i j}^{0}, e_{i j}^{0}, \alpha_{i j}^{0}$ were constants that are related to the properties of materials and $n=3$ [37]. The values of material properties are assumed as follows:

$$
\begin{aligned}
& c_{11}^{0}=15 \mathrm{GPa}, \quad c_{12}^{0}=c_{13}^{0}=6.6 \mathrm{GPa}, \quad c_{33}^{0}=12 \mathrm{GPa}, \\
& c_{44}^{0}=4.4 \mathrm{GPa}, \quad e_{15}^{0}=1.14 \mathrm{C} / \mathrm{m}^{2}, \quad \alpha_{15}^{0}=550 \mathrm{~N} / \mathrm{A} \mathrm{m} .
\end{aligned}
$$

Bone is usually subjected to pressure, and greater pressure can strengthen it. It has been reported that osteoclasts migrate to the positive electrode in an electric field, whereas osteoblasts migrate to the negative electrode [38]. Therefore, all the remodeling rate coefficients are negative. They can be shown as

$$
C_{r r}=-0.06 \mathrm{~m} / \text { day, } \quad C_{\theta \theta}=-0.03 \mathrm{~m} / \text { day }, \quad C_{z z}=-0.04 \mathrm{~m} / \text { day, }
$$

$C_{z r}=-0.01 \mathrm{~m} /$ day, $\quad C_{r}=3 \times 10^{-7} \mathrm{~m} /$ day, and $G_{r}=4 \times 10^{-8} \mathrm{~m} /$ day. 
Table 1 Model state variables and constants

\begin{tabular}{|c|c|c|}
\hline & State variables & \\
\hline$\overline{c_{i j}}$ & Elastic modulus & \\
\hline$e_{i j}$ & Piezoelectric constants & \\
\hline$\alpha_{i j}$ & Piezomagnetic constants & \\
\hline$p$ & Porosity & \\
\hline$N_{R}$ & Number of resorbing BMUs & \\
\hline$N_{R}^{1}$ & Number of resorbing BMUs due to microdamage & \\
\hline$N_{F}^{n}$ & Number of refilling BMUs in internal bone remodeling & \\
\hline$N_{F}^{0}$ & Number of refilling BMUs & \\
\hline$N_{0}^{r}$ & Maximum number of refilling BMUs consumed by internal bone remodeling & \\
\hline$\varepsilon_{i j}$ & Strains & \\
\hline$E_{i}$ & Electrical field & \\
\hline$H_{i}$ & Magnetic field & \\
\hline$\Phi$ & Environmental stimulus & \\
\hline$a$ & Radius of inner surface & \\
\hline$b$ & Radius of outer surface & \\
\hline$\varepsilon$ & Variation rates of inner surface & \\
\hline \multirow[t]{2}{*}{$\eta$} & Variation rates of outer surface & \\
\hline & Constant & Value in this study \\
\hline$Q_{R}$ & Resorbing rate of bone tissues & $2.0 \times 10^{-4}$ \\
\hline$Q_{F}$ & Refilling rate of bone tissues & $1.0 \times 10^{-6}$ \\
\hline$N_{R(\max )}^{1}$ & Maximum BMUs that can be generated by the body & 0.8 \\
\hline$k_{f}$ & Correlation coefficient of the refilling and resorbing process & 1.8 \\
\hline$k_{r}$ & Activation frequency dose-response coefficient & -1.6 \\
\hline$k_{s}$ & Proportional coefficient & 0.6 \\
\hline$k_{e}$ & Inner surface bone remodeling rate coefficient & 0.25 \\
\hline$k_{p}$ & Outer surface bone remodeling rate coefficient & 0.4 \\
\hline$R_{L}$ & Mechanical loading rate & 3000 \\
\hline$f_{e}$ & Frequency of the electromagnetic field & 2 \\
\hline$\Phi_{U}$ & Upper threshold of bone remodeling & 0.0048 \\
\hline$N_{R}^{0}$ & Number of naturally timeworn osteocytes & 0.4 \\
\hline$c_{1}$ & Value of $k_{f}$ during the remodeling process & $3.6 \times 10^{2}$ \\
\hline
\end{tabular}

The initial inner and outer radii are assumed to be

$$
a_{0}=25 \mathrm{~mm}, \quad b_{0}=35 \mathrm{~mm} .
$$

Four loading cases will be illustrated to examine the influence of axial pressure, transverse pressure, electrical field, and magnetic field on bone remodeling. The results are presented in the following.

\subsection{The effect of axial pressure on the bone remodeling process}

To illustrate the influence of axial pressure on bone remodeling, the variation rate of the radii of the inner surface and the changes of the porosity of the bone material are calculated, and are shown in Figs. $2 \mathrm{a}$ and $\mathrm{b}$. The applied loadings are $P=1.6,1.8,2.0,2.2,2.4 \mathrm{kN}$; no other loadings are applied.

Figures $2 \mathrm{a}$ and $\mathrm{b}$ show that axial pressure can strengthen bone tissue by the deposition of new bone material in and on the surface of the bone. Fig. 2a shows the influence of axial pressure on internal bone remodeling. The porosity of bone tissue decreases when the environmental stimuli exceed the MESm, which is defined as the remodeling threshold. Overloads make the bone material denser and stronger. The elastic modulus also increases, which results in a decrease of strains on the bone structure. The environmental stimulus decreases at the same time that the strains become smaller. When the environmental stimulus returns to the remodeling threshold, the porosity will not change any further. This shows that bone tissue can model itself to force the environmental stimulus to revert to the remodeling range. However, when the axial loading is sufficiently high, a further increase will have very little effect on the bone remodeling process. Two factors are responsible for this. One is the lack of osteoclasts. The capacity of the body to produce osteoclasts restricts the upper limit of growth factors. Therefore, the axial loading that can effectively stimulate bone remodeling must have an upper limit. The other factor is saturation of growth factors. This seems to contradict the former factor. Nevertheless, as mentioned above, when the porosity of the bone is sufficiently reduced, the quantity of growth factors will 

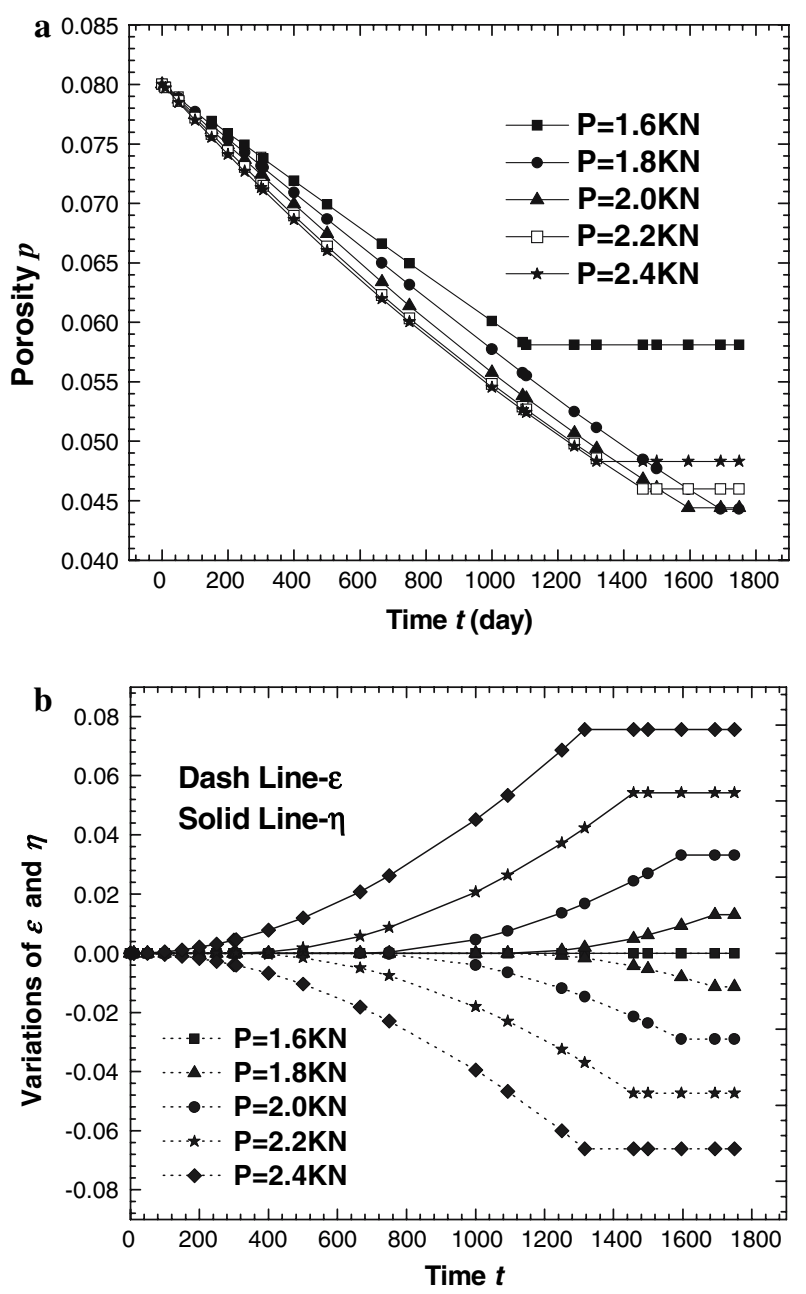

Fig. 2 a Variation of the porosity $p$ for several axial overloads. b Variations of $\varepsilon$ and $\eta$ for several axial overloads

exceed the capacity of the bone to consume them. The unconsumed growth factors are then transported to the surface by the fluid flow. At that time, an increase in the environmental stimulus can only accelerate surface bone remodeling, not internal remodeling. Thus, the internal remodeling rate will also have an upper threshold.

Fig. $2 \mathrm{~b}$ shows the influence of the axial pressure on surface bone remodeling. The results indicate that the inner surface of the bone decreases and the outer surface increases when the axial loadings exceed the MESm. The effect of axial pressure on the inner and outer surfaces indicates that axial pressure can increase the cross-sectional area of the bone and, consequently, a thicker and stronger bone structure can be obtained, which can decrease the strain on the bone structure. Furthermore, greater pressure can result in a greater change in the surface of the bone and accelerate the recovery of injured bone.

If we examine the two figures together, interesting results can be found. In the case of internal bone remodeling, when axial pressure increases, the porosity initially increases and then decreases as another equilibrium state is finally achieved. The remodeling rate (the slope of the line) does not change with time when $P=1.6 \mathrm{kN}$. In other cases, the remodeling rate decreases with the remodeling process. Nevertheless, the surface remodeling rate increases during the remodeling process. The Surface remodeling finally reaches an equilibrium state at the same time as the internal remodeling, although the time at which the surface remodeling process begins is different. The greater the axial loading, the earlier the onset of surface remodeling. These results can be illustrated as follows. We can see that surface bone remodeling is not triggered because of a comparative insufficiency of the growth factors that are generated by the body when $P=1.6 \mathrm{kN}$. Therefore, the internal bone remodeling rate remains unchanged. However, as the environmental stimulus increases, more growth factors are secreted and finally exceed the consumption capacity of internal bone remodeling. 

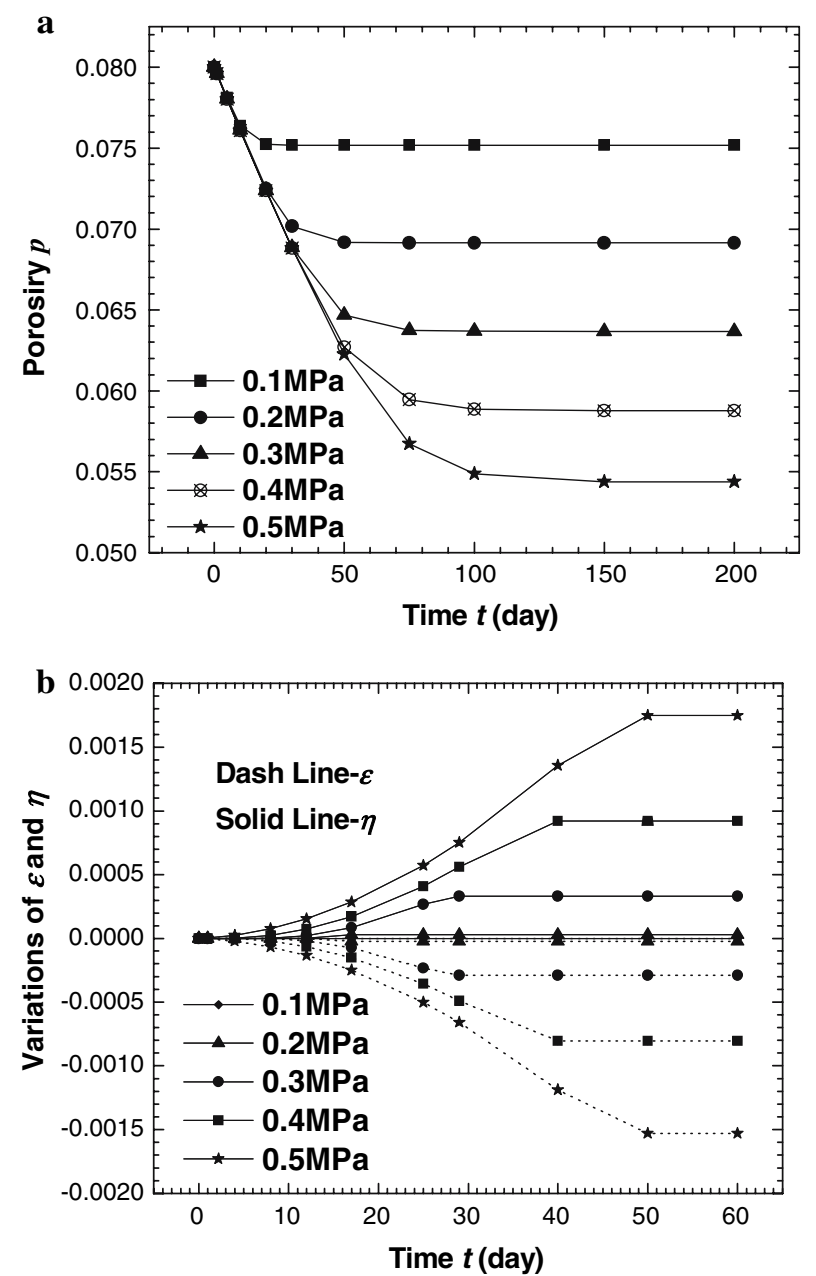

Fig. 3 a Variation of the porosity $p$ for several transverse pressures. b Variations of $\varepsilon$ and $\eta$ for several transverse pressures

Some of the growth factor is then transported to the bone surface, where new bone material is deposited and surface remodeling is triggered, which results in a decrease of the rate of internal bone remodeling. As the porosity of the bone structure decreases, more growth factors are transported to the bone surface. Hence, the internal bone remodeling rate decreases as the surface rate increases, and they finally cease at the same time when the remodeling process reaches equilibrium. This is different from the following cases because of the inhomogeneity of the environmental stimulus. In addition, if the environmental stimulus increases, more growth factors are released into the bone fluid. It is obvious that saturation can then more easily be reached and surface remodeling be triggered earlier. Coupled remodeling is obviously more effective than separate remodeling. The final porosity is then increased as the environmental stimulus increases. Bone tissue seems to have a capacity to prevent the bone structure from becoming so dense that bone fluid cannot be transported effectively. In this paper we are unable to state the exact threshold; further theoretical and experimental investigation is needed.

\subsection{The effect of transverse pressure on the bone remodeling process}

In this section we consider the influence of transverse loads on the bone remodeling process. The results are shown in Figs. 3a and b. The applied loadings are $P=1.2 \mathrm{kN}, p_{t}=0.1,0.2,0.3,0.4,0.5 \mathrm{MPa}$; no other loadings are applied.

It can be seen from Figs. 3a and $b$ that transverse pressures have some similar effects on the bone remodeling process as those of axial loadings. For example, they can make the bone structure stronger and denser. They can also increase the cross-sectional area of bone and make it thicker. Moreover, the rate of change increases 
as the magnitude of the loading increases. For surface remodeling, a difference in the trigger time also exists. However, as noted, other differences are evident. First, for internal bone remodeling, a gradually convergent process can be observed compared to the former case; for surface remodeling, the rate does not increase from the beginning to the end of the remodeling process, rather it first increases and subsequently decreases. The time cost for internal bone remodeling also varies from that for surface remodeling. Surface remodeling takes less time than internal remodeling.

These differences result from the inhomogeneity of the environmental stimulus. As the transverse pressure is loaded, an inhomogeneous strain field is generated, which results in the inhomogeneous distribution of the environmental stimulus. The quantity of growth factor released into the bone fluid then varies in different areas of the bone structure. As the environmental stimulus decreases due to the bone remodeling process, the inhomogeneity disables the bone tissues in different areas of the bone structure from reaching the MESm at the same time. This means that the bone tissue in some areas of the bone cannot excrete excess growth factor (i.e., redundant growth factors after the refilling process) to deposit additional bone material because the environmental stimulus is within the remodeling range. However, additional growth factors can still be generated in the remaining areas at the same time, and new bone material deposition will continue until all of the bone tissues return to the remodeling mode. The amount of bone tissue in the remodeling state will then diminish as the bone remodeling process goes on. Thus, the gradual divergence of internal bone remodeling and the rate change of surface remodeling can be easily understood. The reversal of the surface remodeling rate occurs when some of the bone tissue reverts to the remodeling mode. In the case of axial loading, the environmental stimulus is distributed homogeneously, which means that all of the bone tissue can revert to the remodeling mode at the same time and internal and surface remodeling cease simultaneously. However, in this case, when surface remodeling ceases not all of the bone tissue stops excreting additional growth factor. Internal bone remodeling will continue until all of the bone tissue is in the remodeling mode. Thus, the surface and internal remodeling cease at different times. It should be mentioned that, as we assumed above, growth factors can be homogeneously distributed in bone structure due to the flow of bone fluid, so that a homogeneous bone structure is maintained.

\subsection{The effect of an electromagnetic field on the bone remodeling process}

As earlier investigations have shown that the influence of electrical and magnetic fields is similar [16,17], we here consider only the electrical field. The results are shown in Figs. 3a and b. The applied loadings are $P=1.2 \mathrm{kN}, \varphi=5,10,15,20,25 \mathrm{~V}, f_{e}=2 \mathrm{~Hz}$, and no other loadings are applied; here, $\varphi=\varphi_{b}-\varphi_{a}$.

Figures $4 \mathrm{a}$ and $\mathrm{b}$ show the effect of electrical loading on the internal and surface bone remodeling processes. It can be seen that, as the electrical loading increases to a certain level, bone remodeling can be triggered. An electrical field is able to model the bone structure to fit its environment so that the loading does not break or harm it. Electrical fields can also make bone structure stronger and denser, and increase the cross-sectional area of bone and make it thicker. The rates of change increase as the intensity of the loading increases. All these features are effective methods to return a remodeling bone structure to the remodeling mode. Furthermore, gradual divergence of the internal remodeling rate, differences in the trigger time, reversal of the change of the rate of surface remodeling, and different cessation times can also be seen in this case due to the inhomogeneous distribution of the environmental stimulus, as in the case of transverse pressure. However, an electrical field seems to affect bone remodeling more moderately than does transverse pressure. The changes in the remodeling rate under the electrical field are smoother and less marked.

\subsection{The effect of multifield loadings on the bone remodeling process}

The results in this section show bone remodeling behavior under multifield loadings. This case simulates a bone structure that is subjected to axial and transverse pressure and a pulsed magnetic field. Several magnetic loadings of different intensity are examined. The results can be seen in Figs. 5a and b. The applied loadings are $P=1.5 \mathrm{kN}, p_{t}=0.2 \mathrm{MPa}, \psi=-0.2,-0.1,0,0.1,0.2 \mathrm{~A}, f_{e}=2 \mathrm{~Hz}$; here, $\varphi=\varphi_{b}-\varphi_{a}$.

Figures $5 \mathrm{a}$ and $\mathrm{b}$ show that a positive magnetic field can stimulate the bone remodeling process whereas a negative magnetic field can do the opposite. When the bone structure is loaded by a positive magnetic field, both the density and cross-sectional area increase, and stronger and thicker bone is generated. The more intense the magnetic field, the stronger and thicker the bone, but a negative magnetic field can weaken bone remodeling, making bone thinner and more porous. A positive magnetic field can play the same role as an increase in 

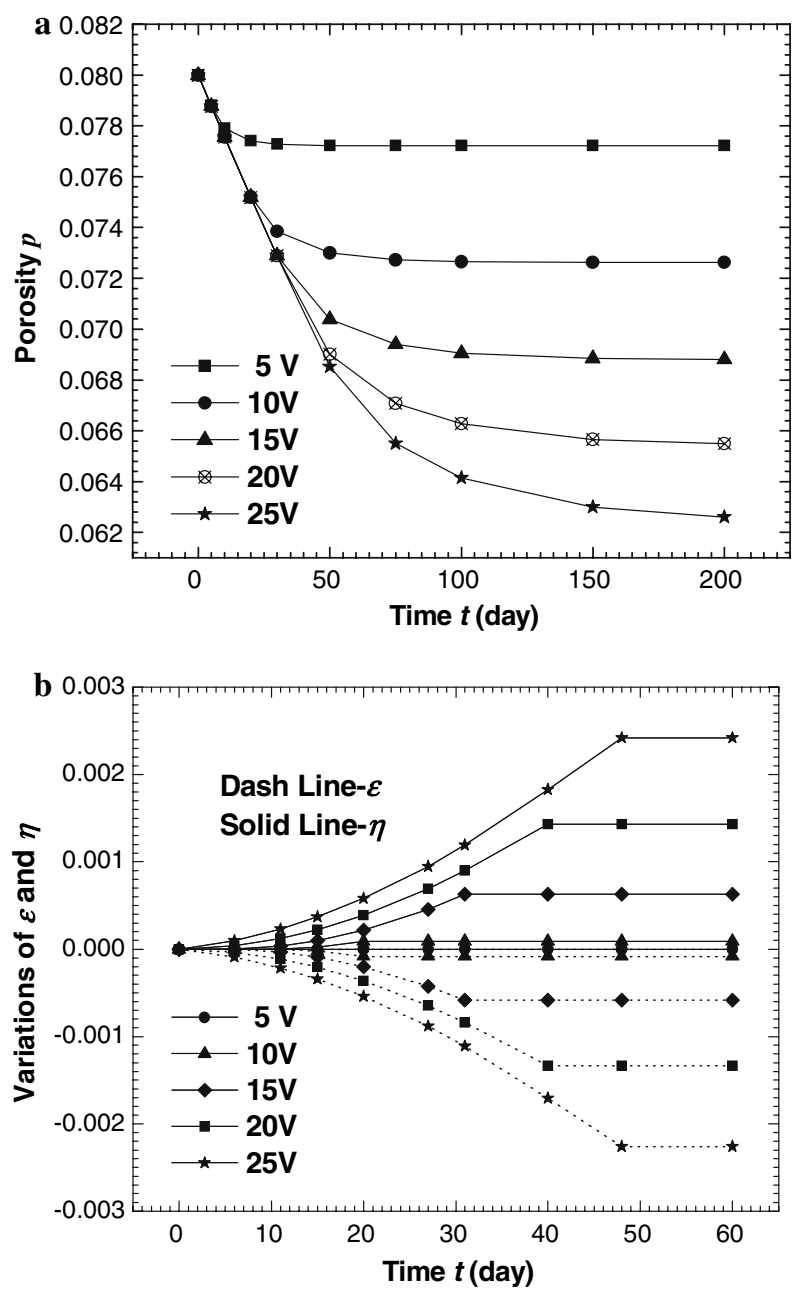

Fig. 4 a Variation of the porosity $p$ subjected to different electrical fields. b Variations of $\varepsilon$ and $\eta$ subjected to different electrical fields

transverse loading, whereas a negative magnetic field can replicate the effect of a decrease in such loading. However, the effect of magnetic loading is smooth and gentle. Used as a method to control remodeling, it is therefore an appropriate way of dealing with bone conditions.

\section{Conclusions}

In this paper we propose a growth-factor-related model to simulate bone surface remodeling behavior under an electromagnetic field when the bone is overloaded. Among theoretical models for bone remodeling and remodeling, there are some notable features [39]. For example, because more than one hypothesis can explain most collections of facts, people can reasonably conceive different explanations for the same data. For this reason, our model is different from others and the results appear unique. It is harder to validate hypotheses than the facts that are their foundation. Despite these difficulties, however, we plan to seek experimental validation of this model. Traditionally, many good hypotheses have proved more useful than the data that originally led to the development of these hypotheses. Supplementing early views about bone physiology with newer evidence, ideas, and terminology led to a new concept of bone physiology. Our model is based on observations of the biological effects of extremely low-frequency electromagnetic fields. The surface remodeling behavior is simulated numerically, based on the proposed model, and the influences of axial pressure, transverse pressure, and electromagnetic fields are examined. The results suggest interesting elements of bone physiology, which may be useful for clinical practice. For example, the axial and transverse pressure or electromagnetic loadings 

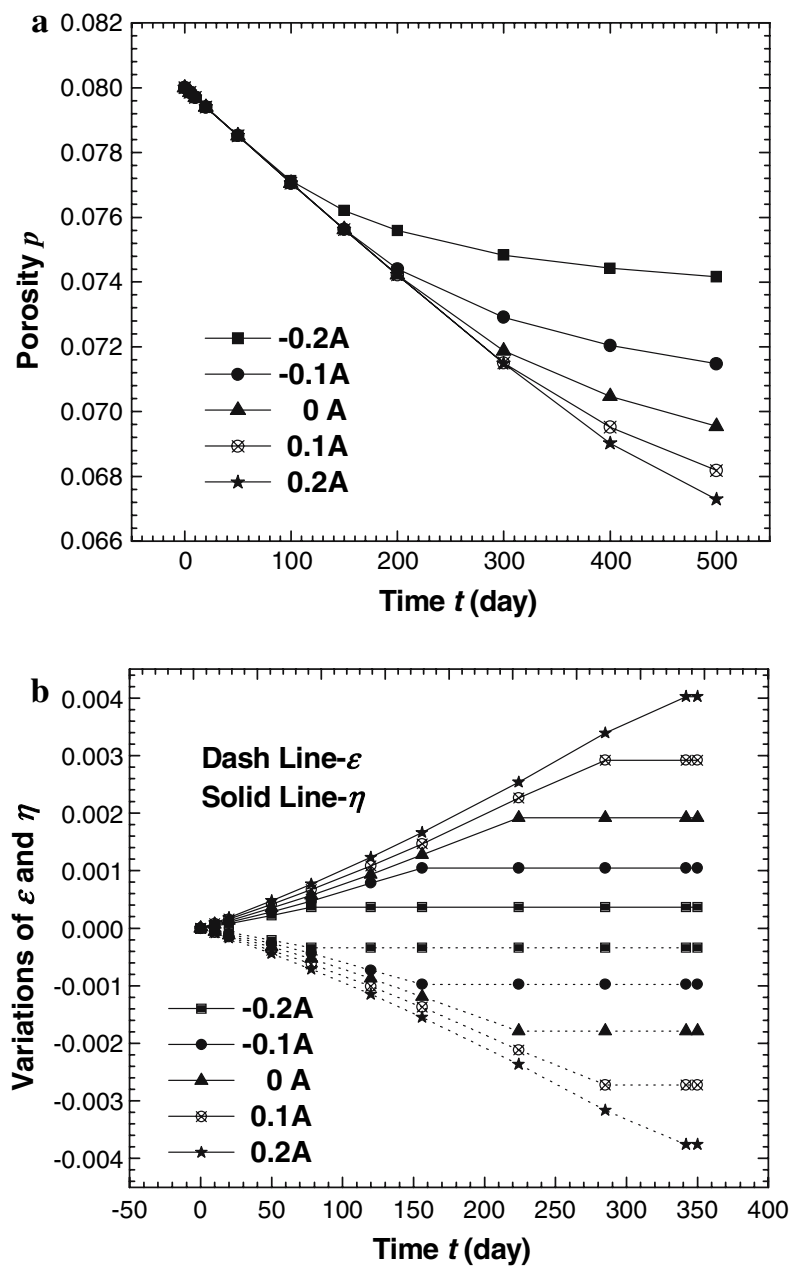

Fig. 5 a Variations of the porosity $p$ for several different multifield loadings. b Variations of $\varepsilon$ and $\eta$ for several different multifield loadings

can really stimulate the bone remodeling and accelerate the bone recovery process. Our results also suggest a potential application of those method in the medical treatment of osteoporosis, tendonitis, osteonecrosis, fractures, and nonunion, etc. However, it should be mentioned that our results are obtained by theoretical simulation. The concrete applications are not indicated based on present results. Furthermore, theoretical and experimental investigation is necessary to supplement the present model to refine and validate it before it can be applied to direct the medical treatment of these bone diseases.

Acknowledgment The work described in this paper was fully supported by a grant from City University of Hong Kong (Project No. 7002076).

\section{References}

1. Bessett, C.A., Valdes, M.G., Hernandez, E.: Modification of fracture repair with selected pulsing electromagnetic fields. J. Bone Joint Surg. 64, 888-895 (1982)

2. Mcleod, K.J., Rubin, C.T.: The effect of low-frequency electrical fields on osteogenesis. J. Bone Joint Surg. 74, 920-929 (1992)

3. Giordano, N., Battisti, E.: Effect of electromagnetic fields on bone mineral density and biochemical markers of bone turnover in osteoporosis: a single-blind, randomized pilot study. Curr. Ther. Res. 62, 187-193 (2001)

4. Frost, H.M.: Changing concepts in skeletal physiology: Wolff's Law, the Mechanostat, and the "Utah Paradigm." Am. J. Hum. Biol. 10, 599-605 (1998)

5. Frost, H.M.: Dynamics of bone remodeling. In: Frost, H.M. (ed.) Bone biodynamics. Little, Brown and Co., Boston (1964) 
6. Cowin, S.C., Hegedus, D.M.: Bone remodeling I: Theory of adaptive elasticity. J. Elasticity 6, 313-326 (1976)

7. Hegedus, D.H., Cowin, S.C.: Bone remodeling II: Small strain adaptive elasticity. J. Elasticity 6, 337-352 (1976)

8. Cowin, S.C., Nachlinger, R.R.: Bone remodeling III: Uniqueness and stability in adaptive elasticity theory. J. Elasticity 8, 285-295 (1978)

9. Martin, R.B.: A mathematical model for fatigue damage repair and stress fracture in osteonal bone. J. Orthop. Res. 13, 309-316 (1995)

10. Beaupré, G.S., Orr, T.E., Carter, D.R.: An approach for time-dependent bone remodeling and remodeling: theoretical development. J. Orthop. Res. 651-661 (1990a)

11. Beaupré, G.S., Orr, T.E., Carter, D.R.: An approach for time-dependent bone remodeling and remodeling: Application: A preliminary remodeling simulation. J. Orthop. Res. 8, 662-670 (1990b)

12. Gjelsvik, A.: Bone remodeling and piezoelectricity-I. J. Biomech. 6, 69-77 (1973a)

13. Gjelsvik, A.: Bone remodeling and piezoelectricity-II. J. Biomech. 6, 187-197 (1973b)

14. Qin, Q.H., Ye, J.Q.: Thermolectroelastic solutions for internal bone remodeling under axial and transverse loads. Int. J. Solids and Struct. 41, 2447-2460 (2004)

15. Qin, Q.H., Qu, C.Y., Ye, J.Q.: Thermolectroelastic solutions for surface bone remodeling under axial and transverse loads. Biomaterials 26, 6798-6810 (2005)

16. Qu, C.Y., Qin, Q.H., Kang, Y.L.: Thermomagnetoelectroelastic prediction of the bone surface remodeling under axial and transverse loads. In: Proceedings of the 9th International Conference on Inspection, Appraisal, Repairs \& Maintenance of Structures, 373-380, Fuzhou, China, October 20-21 (2005)

17. Qu, C.Y., Qin, Q.H., Kang, Y.L.: A hypothetical mechanism of bone remodeling and remodeling under electromagnetic loads. Biomaterials 27, 4050-4057 (2006)

18. Fukada, E., Yasuda, I.: On the piezoelectric effect of bone. J. Physiol. Soc. Jpn. 12, 1158-1162 (1957)

19. Frost H.M.: The mechanostat: a proposed pathogenic mechanism of osteoporosis and the bone mass effects of mechanical and nonmechanical agents. Bone 2, 73-85 (1987)

20. Frost, H.M.: Bone's mechanostat: A 2003 update. Anat. Rec. 275A, 1081-1101 (2003)

21. Frost, H.M.: Structural adaptations to mechanical usage (SATMU): 1. Redefining Wolff's Law: The bone remodeling problem. Anat. Rec. 226, 403-413 (1990)

22. Rubin, C.T., Lanyon, L.E.: Regulation of bone mass by mechanical strain magnitude. Calcified. Tissue Int. 37, 411-417 (1985)

23. Jee, W.S.S., Li, X.J., Ke, H.Z.: Skeletal adaptations to mechanical usage in the rat. Cells. Mat. S1, 131-142 (1991)

24. Batra, G.S., Hainey, L., Freemont, A.J., Andrew, G., Saunders, P.T., Hoyland, J.A., Braidman, I.P.: Evidence for ellspecific changes with age in expression of oestrogen receptor (ER) alpha and beta in bone fractures from men and women. J. Pathol. 200, 65-73 (2003)

25. Oursler, M.J., Kassem, M., Turner, R., Riggs, B.L., Spelsberg, T.C.: Regulation of bone cell function by gonadal steroids. In: Marcus, R., Feldman, D., Kelsey, J. (eds.) Osteoporosis, pp. 237-260. Academic, San Diego (1996)

26. Skerry, T.M., Bitensky, L., Chayen, J., Lanyon, L.E.: Early strain-related changes in enzyme activity in osteocytes following bone loading in vivo. J. Bone Miner. Res. 4, 783-788 (1989)

27. Klein-Nulend, J., Van der Plas, A., Semeins, C.M., Ajubi, N.E., Frangos, J.A., Nijweide, P.J., Burger, E.H.: Sensitivity of osteocytes to biomechanical stress in vitro. FASEB J. 9, 441-445 (1995)

28. Bakker, A., Klein-Nulend, J., Burger, E.: Shear stress inhibits while disuse promotes osteocyte apoptosis. Biochem. Bioph. Res. 320, 1163-1168 (2004)

29. Mullender, M.G., Huiskes, R.: A proposal for the regulatory mechanism of Wolff's law. J. Orthop. Res. 13, 503-512 (1995)

30. Nielsen, H.M., Andreassen, T.T., Leder, T.: Local injection of TGF- $\beta$ increasing the strength of tibial fracture in the rat. Acta. Orthop. Scand. 65(1), 37-41 (1994)

31. Asahina, J., Waranabe, M., Sakurai, N.: Repair of bone defect in primate mandible using a bone morphogenetic protein (BMP)-hydroxyapatite-collagen composite. J. Med. Dent. Sci. 44(3), 63-70 (1997)

32. Weinreb, M., Suponiyzky, I., Keila, S.: Systematic administration of an anabolic dose of $\mathrm{PGE}_{2}$ in young rats increases the osteogenic capacity of bone marrow. Bone 20(6), 521-526 (1997)

33. Lammens, J., Liu, Z., Aerssans, J.: Distraction bone healing versus osteotomy healing: A comparative biochemical analysis. J. Bone. Miner. Res. 13(2), 279-286 (1998)

34. Fitzsimmons, R.J., Strong, D.D., Mohan, S.: Low-amplitude, low-frequency electric field-stimulated bone cell proliferation may in part be mediated by increased IGF-I release. J. Cell Physiol. 150(1), 84-89 (1992)

35. Nagai, M., Ots, M.: Pulsing electromagnetic fields stimulates mRNA expression of bone morphogenetic protem-2 and -4 . J. Dent. Res. 73(10), 1601-1605 (1994)

36. Zhung, H.M., Wei, W., Seldes, R.M.: Electrical stimulation induces the level of TGF- $\beta$ mRNA in osteoblastic cells by a mechanism involving a calcium/ calmodulin pathway. Biochem. Bioph. Res. 237(2), 225-229 (1997)

37. Currey, J.D.: The effect of porosity and mineral content on the Young's modulus of elasticity of compact bone. J. Biomech. 21, 131-139 (1988)

38. Hillsley, M.V., Frangos, J.A.: Bone tissue engineering: The role of interstitial fluid flow. Biotechnol. Bioeng. 43, 573-581 (1994)

39. Frost, H.M.: Emerging views about "osteoporosis," bone health, strength, fragility, and their determinants. J. Bone. Miner. Metab. 20, 319-325 (2002) 